\title{
DISSOLUTION OF THE DILEMMA OR CIRCULATION PROBLEM USING THE ANALYTIC NETWORK PROCESS
}

\author{
Toshimasa Ozaki * \\ Nagoyagakuin University \\ Nagoya, Aici, Japan \\ E-mail: ozaki@ngu.ac.jp \\ Shin Sugiura \\ Meijo University \\ Kani, Gifu,Japan \\ E-mail: shinsu@urban.meijo-u.ac.jp \\ Eizo Kinoshita \\ Meijo University \\ Kani, Gifu,Japan \\ E-mail: kinoshit@urban.meijo-u.ac.jp
}

\begin{abstract}
This paper presented the new method of dissolution of the dilemma or circulation problem by the ANP. The super matrix of the ANP both with the inverse matrix of the dilemma or circulation matrix and with the criteria matrix was considered. The paper showed mathematically that the eigenvector of the super-matrix serves as a solution of the dilemma or circulation problem. Moreover, the descriptive interpretation of the proposal method was performed.
\end{abstract}

Keywords: super matrix, dilemma or circulation problem, eigenvector, fallacy of composition

\section{Introduction}

There are many cases with dilemma or circulation problem where the best choice cannot be obtained based on transitive relation. Especially, the activity of the enterprise of this problem is not unusual. For instance, let's think about the product planning that draws up customer's needs based on the conception of the reversal of the value chain. In this case, the dissension and the confrontation are often seen between

\footnotetext{
${ }^{*}$ Corresponding author
} 
the production department near the customer, the development and the management department in a strategic standpoint. That is, there emerges an evaluation problem with the dilemma.

Arrow $^{(1)}(1951)$ proved that irrationality is generated in the society where the evaluator is composed of multiple types of people in the general possibility theorem whenever a social decision making is done from more than three choices. Such irrationality is often generated by the difference in the standpoint where each evaluator is left. So the decision-making technique to the evaluation problem with the dilemma or circulation is extremely important to the definition of Simon saying "To manage is to decide to make ".

Because the AHP is one technique of the decision-makings and it requires the transitive relation, the influence of dilemma is taken into adjustment level C.I, and has been treated as a decision making stress problem. In a large close-up of the evaluation problem with dilemma in full scale, the research of the reversal problem of the order in the AHP is like the opportunity. Because the reversal of the order in the decision-making method becomes a fatal fault of the technique, Sugiura,S. \& Kinoshita,E.(2005) showed the dissolution of the evaluation problem with dilemma or circulation by using the Concurrent Convergence $^{(3)}$. However, the dissolution method by the ANP has not been proposed yet. This paper describes a dissolution method of the dilemma or circulation problem by using the ANP and some findings to the ANP.

\section{Dissolution method of the dilemma or circulation problem}

\subsection{Previous method}

The decision making determines man's subsequent behavior. Therefore, the method of the problem should offer the decision-maker preferable information on the alternatives and risk information according to the selection. However, there is no decision-making method that gives significant information on the evaluation problem with circulative, for instance, " Rock> Scissor", "Scissor> Rock ", " Rock > Paper ".

Sugiura,S. \& Kinoshita,E. (2005) classified the circulation problems into two kinds as following, Simple Circulation and Circulation by fallacy of composition, and showed the unified solution. When the alternatives are evaluated by a specific basis of selection, Simple Circulation is generated. Therefore, the rock-paper- scissors becomes Simple Circulation. When two or more decision-makers exist, and when decision maker's standpoint are different, Circulation by fallacy of composition is generated.

The dilemma or circulation problem in the AHP was taken up as an architectural issue by E.Triantapyllou and by E.Kinoshita et al, with comparing two alternatives for the cancellation of the order reversal problem. For instance, three types of Japanese Sinkansen: "Kodama","Hikari", and "Nozomi" are assumed to be mutual alternatives, and "Amenity (C1)" and "Economy (C2)" are assumed to be a criterion in the illustration of Simple Circulation that Sugiura,S. \& Kinoshita,E. (2005). ${ }^{(2)}$ set. In this example, is inferred the order of "Nozomi">"Kodama">"Hikari", because "Kodama" (0.68)> "Hikari" (0.32) and "Nozomi" (0.6) > "Kodama" (0.4) has been approved when "Amenity (C1)" and "Economy 
(C2)" are assumed to be a criterion. But, it is impossible to set priorities between three alternatives because of "Hikari" (0.55)> "Nozomi" 0.45). (Table 1.)

Table.1. Illustration of Simple Circulation

\begin{tabular}{c|c|c|c}
\hline & C1(0.8) & C2(0.2) & Value \\
\hline Kodama & 0.7 & 0.6 & 0.68 \\
\hline Hikari & 0.3 & 0.4 & 0.32 \\
\hline & $\mathrm{C} 1(0.5)$ & $\mathrm{C} 2(0.5)$ & Value \\
\hline Nozomi & 0.9 & 0.3 & 0.6 \\
\hline Kodama & 0.1 & 0.7 & 0.4 \\
\hline & $\mathrm{C} 1(0.3)$ & $\mathrm{C} 2(0.7)$ & Value \\
\hline Hikari & 0.2 & 0.7 & 0.55 \\
\hline Nozomi & 0.8 & 0.3 & 0.45 \\
\hline
\end{tabular}

The element not evaluated is inferred, and the evaluation by final AHP becomes the following. ${ }^{(2)}$

Table 2. Pair comparison

\begin{tabular}{|l|c|c|c||c|}
\cline { 2 - 5 } \multicolumn{1}{c|}{} & Kodama & Hikari & Nozomi & Eigen value \\
\hline Kodama & 1 & 2.1250 & 0.6667 & 0.3712 \\
\hline Hikari & 0.4706 & 1 & 1.2222 & 0.2749 \\
\hline Nozomi & 1.5000 & 0.8182 & 1 & 0.3539 \\
\hline
\end{tabular}

In the Concurrent Convergence by Sugiura \& Kinoshita, they put it with

$$
\begin{aligned}
& {\left[\begin{array}{ccc}
1 & 2.1250 & 0.6667 \\
0.4706 & 1 & 1.2222 \\
1.5000 & 0.8182 & 1
\end{array}\right]=\left[\begin{array}{lll}
M_{1}^{X X} & M_{2}^{Y X} & M_{3}^{Z X} \\
M_{1}^{X Y} & M_{2}^{Y Y} & M_{3}^{Z Y} \\
M_{1}^{X Z} & M_{2}^{Y Z} & M_{3}^{Z Z}
\end{array}\right]} \\
& {\left[\begin{array}{l}
\sqrt[3]{M_{1}^{X X} \cdot M_{2}^{X X} \cdot M_{3}^{X X}} \sqrt[3]{M_{1}^{Y X} \cdot M_{2}^{Y X} \cdot M_{3}^{Y X}} \sqrt[3]{M_{1}^{Z X} \cdot M_{2}^{Z X} \cdot M_{3}^{Z X}} \\
\sqrt[3]{M_{1}^{X Y} \cdot M_{2}^{X Y} \cdot M_{3}^{X Y}} \sqrt[3]{M_{1}^{Y Y} \cdot M_{2}^{Y Y} \cdot M_{3}^{Y Y}} \sqrt[3]{M_{1}^{Z Y} \cdot M_{2}^{Z Y} \cdot M_{3}^{Z Y}} \\
\sqrt[3]{M_{1}^{X Z} \cdot M_{2}^{X Z} \cdot M_{3}^{X Z}} \sqrt[3]{M_{1}^{Y Z} \cdot M_{2}^{Y Z} \cdot M_{3}^{Y Z}} \sqrt[3]{M_{1}^{Z Z} \cdot M_{2}^{Z Z} \cdot M_{3}^{Z Z}}
\end{array}\right]=\left[\begin{array}{ccc}
1 & 1.3505 & 1.0490 \\
0.7405 & 1 & 0.7768 \\
0.9533 & 1.2874 & 1
\end{array}\right]}
\end{aligned}
$$

the matrix is obtained ${ }^{(3)}$. All eigenvectors are thoroughly requested from one as for the ratio of the evaluation of final alternatives when regularizing it, and this result showed that the priority level is uniquely decided.

\subsection{Proposed method}

Some information needs AHP about all alternatives or ratios of the evaluation item usually. However, the paired comparison might be difficult to evaluate it. For instance, let's regard the circulative matrix in Table 1.

$$
\boldsymbol{U}=\left[\begin{array}{ccc}
a_{1} & b_{1} & 0 \\
a_{2} & 0 & c_{1} \\
0 & b_{2} & c_{2}
\end{array}\right]
$$


Each element of a couple of comparison matrix shown by $A, B$ and $C$ a as mentioned above.

$$
\boldsymbol{U}_{A H P}=\left[\begin{array}{lll}
a_{1} / a_{1} & a_{1} / a_{2} & b_{1} / b_{2} \\
a_{2} / a_{1} & a_{2} / a_{2} & c_{1} / c_{2} \\
b_{2} / b_{1} & c_{2} / c_{1} & c_{2} / c_{2}
\end{array}\right]=\left[\begin{array}{ccc}
1 & A & B \\
1 / A & 1 & C \\
1 / B & 1 / C & 1
\end{array}\right]
$$

Let's think about the ANP that sets these two matrixes by assuming the matrix that interpolates the loss of the evaluation value to be $\boldsymbol{W}$. For instance, the element not evaluated is assumed to be 0 and the evaluated element are assumed to be 1 . Then the super-matrix of the ANP will be made from the criteria matrix.

$$
\left[\begin{array}{cc}
\boldsymbol{0} & \boldsymbol{W} \\
\boldsymbol{U} & 0
\end{array}\right]=\left[\begin{array}{cccccc}
0 & 0 & 0 & 1 & 1 & 0 \\
0 & 0 & 0 & 1 & 0 & 1 \\
0 & 0 & 0 & 0 & 1 & 1 \\
0.68 & 0.4 & 0 & 0 & 0 & 0 \\
0.32 & 0 & 0.55 & 0 & 0 & 0 \\
0 & 0.6 & 0.45 & 0 & 0 & 0
\end{array}\right]
$$

The definition of this criterion matrix $\boldsymbol{W}$ is insufficient. The eigenvector of $\boldsymbol{U} \boldsymbol{W}$ is $(0.3620,0.2778,0.3602)$, the order is maintained, and the error margin with each eigenvector is within \pm 0.01 in compared with the value of Table 2. Therefore, we may obtain the same solution as Eq.(2) by the ANP, if suitable criterion matrix $\boldsymbol{W}$ is defined. Then, we think about the eigenvector of the supermatrix $\boldsymbol{U}$ based on the evaluation matrix $\boldsymbol{W}$ whose element without the evaluation is 0 , by taking the matrix inverse of the dilemma or circulation .

$$
\boldsymbol{W}=\left[\begin{array}{ccc}
\frac{b_{2} c_{1}}{a_{1} b_{2} c_{1}+a_{2} b_{1} c_{2}} & \frac{b_{1} c_{2}}{a_{1} b_{2} c_{1}+a_{2} b_{1} c_{2}} & 0 \\
\frac{a_{2} c_{2}}{a_{1} b_{2} c_{1}+a_{2} b_{1} c_{2}} & 0 & \frac{a_{1} c_{1}}{a_{1} b_{2} c_{1}+a_{2} b_{1} c_{2}} \\
0 & \frac{a_{1} b_{2}}{a_{1} b_{2} c_{1}+a_{2} b_{1} c_{2}} & \frac{a_{2} b_{1}}{a_{1} b_{2} c_{1}+a_{2} b_{1} c_{2}}
\end{array}\right]
$$

The eigenvector to the maximum eigen value $k$ of this matrix is assumed to be $x$ and $z$.

$$
\left[\begin{array}{cc}
\boldsymbol{0} & \boldsymbol{W} \\
\boldsymbol{U} & \boldsymbol{0}
\end{array}\right]\left[\begin{array}{l}
\boldsymbol{x} \\
\boldsymbol{z}
\end{array}\right]=k\left[\begin{array}{l}
\boldsymbol{x} \\
\boldsymbol{z}
\end{array}\right]
$$

The overall judgement of the alternatives is shown by eigenvector $z$ to maximum eigenvalue. Let's compare this eigenvector $z$ and the eigenvector $y$ at value $\alpha$ shown by Eq.(2). 
$y_{1}=\frac{B\{A C(1-\alpha)-B\}}{B(1-\alpha)-A C}$ and $y_{2}=\frac{C\{B(1-\alpha)-A C\}}{A C(1-\alpha)-B}$ are element of the eigenvector is assumed to be $\boldsymbol{y}\left(y_{1}, y_{2}, y_{3}\right)$ and $y_{3}=1$.

On the other hand, the eigenvector of $z$ is written by $1-\frac{1}{1-k^{2}}=\alpha-1$ as $z_{3}=1, z\left(z_{1}, z_{2}, z_{3}\right)$ is eigenvalue of Eq.(5).

$$
\begin{gathered}
z_{1}=\frac{B\left\{(B+A C)\left(1-k^{2}\right)-A C\right\}}{(B+A C)\left(1-k^{2}\right)-B}=\frac{B\{B-(1-\alpha) A C\}}{A C-(1-\alpha) B}=\frac{B\{(1-\alpha) A C-B\}}{(1-\alpha) B-A C}=y_{1} \\
\mathrm{z}_{2}=\frac{C\left\{(B+A C)\left(1-\mathrm{k}^{2}\right)-B\right\}}{(B+A C)\left(1-\mathrm{k}^{2}\right)-A C}=\frac{C\{A C-(1-\alpha) B\}}{B-(1-\alpha) A C}=\frac{C\{(1-\alpha) B-A C\}}{(1-\alpha) A C-B}=\mathrm{y}_{2} .
\end{gathered}
$$

Thus, in spite of $\alpha$ and $k$, the eigenvector of $\boldsymbol{U} \boldsymbol{W}$ is the same as the eigenvector of $\boldsymbol{U}_{A H P}$. That is, $a_{i j}=1 / a_{j i}$ is assumption in the relations between elements of AHP. Therefore, such as a dilemma or circulation problem can be solved by the proposed method by requesting the eigenvector in descriptive ANP to the maximum eigenvalue.

\section{Descriptive interpretation of the proposal method}

It was mathematically shown that Eq.(5) has the same eigenvector as Eq.(2). At the next stage, this method will be defined. The matrix $\boldsymbol{W}$ is

$$
\boldsymbol{W}=\left[\begin{array}{ccc}
w_{11} & w_{12} & 0 \\
w_{21} & 0 & w_{23} \\
0 & w_{32} & w_{33}
\end{array}\right]
$$

$\boldsymbol{U} \boldsymbol{W}$ can be presented as follows.

$$
\boldsymbol{U W}=\left[\begin{array}{ccc}
a_{1} w_{11}+b_{1} w_{21} & b_{1} w_{12} & b_{1} w_{23} \\
a_{2} w_{11} & a_{2} w_{12}+c_{1} w_{13} & c_{1} w_{33} \\
b_{2} w_{21} & c_{2} w_{13} & b_{2} w_{23}+c_{2} w_{33}
\end{array}\right]
$$

Let's think about the action of $\boldsymbol{W}$ on $\boldsymbol{U} \boldsymbol{W}$ in the ANP. Let's imagine the situation that there is demand $\boldsymbol{W}$ from each alternative through the evaluator $T_{1}, T_{2}$ and $T_{3}$ of three people giving the evaluation value indicated in Eq.(1) to the alternative "Kodama", "Hikari" and "Nozomi"

Each line of Eq.(7) is an evaluation value of alternatives, and it is possible to think each row to be an evaluation value of three virtual evaluators $T_{1}, T_{2}$ and $T_{3}$. The ANP method minimizes the difference of the contradiction between alternatives and the criteria. 
For instance, we think about the process of mutual evaluation as an example of alternatives "Kodama". This case where the evaluator evaluates Kodama is divided into the following four cases.

(1)The case that the evaluator $T_{1}$ gives $a_{1}$ to "Kodama" (The main factor is "Kodama")

Naturally, the other alternatives of "Hikari" and "Nozomi"will demand to review evaluation value $a_{1}$. "Hikari" and "Nozomi" start correcting evaluation value to evaluator $T_{3}$ and $T_{2}$. At this time, since it is $b_{2}$ and $c_{1}$ to influence the evaluation of $a_{1}$, these two multiplications as a size of the demand and assume evaluation value $a_{1}$ review $w_{11}$ to be $w_{11}=b_{2} c_{1}$ now.

We may think that multiplication $a_{1} w_{11}$ of evaluation value $a_{1}$ and evaluation value $a_{1} w_{11}$ represents the satisfaction rating of "Kodama". So the evaluation $a_{1}$ of "Kodama" dominates this satisfaction rating, then the main factor of the satisfaction rating is "Kodama".

(2) The case that the evaluator $T_{2}$ gives $b_{1}$ to

"Kodama" (The main factor is "Kodama") : In this case, "Hikari" will demand evaluation value $b_{1}$ correction to the evaluator $T_{1}$ and "Nozomi" will demand evaluation value correction to the evaluator $T_{3}$ too.

When the evaluator $T_{2}$ gives $b_{1}$ to "Kodama", $a_{2}$ and $c_{2}$ influence the evaluation value $b_{1}$. Then, the size of the demand may be cut by $w_{21}=a_{2} c_{2}$, and then $b_{1} w_{21}$ is the satisfaction rating will go up among the evaluators $T_{1}, T_{2}$ and $T_{3}$ to "Kodama". For example, "Kodama" becomes a main factor of the satisfaction rating because $b_{1} w_{21}$ becomes $\left(1-a_{1}\right) b_{1} c_{2}$ when assuming $a_{1}+a_{2}=1$.

(3) The case that the evaluator $T_{2}$ gives $b_{1}$ to

"Kodama" (The main factor is "Hikari")

In this case, the evaluator $T_{1}$ gives $a_{1}$ to "Kodama". At this time, evaluators will evaluate either of "Hikari" or "Nozomi". The evaluator $T_{3}$ only has to evaluate "Nozomi", when the opportunity where "Hikari" receives the evaluation is abandoned. Therefore, there are only $a_{1}$ and $c_{2}$ who can be able to 
place the objection between the evaluation value of this $b_{1}$ when the evaluator $T_{2}$ gives $b_{1}$ to "Kodama". However, when the alternative lodges an objection not only in other evaluation values but also in its own evaluation values, the equilibrium solution cannot be made. Therefore, the equilibrium solution can be created by making the complaint only to the others' evaluations. A satisfaction rating is $b_{1} w_{12}$ and leads to $b_{1} w_{12}=\left(1-a_{2}\right) b_{1} c_{2}$. That is, "Hikari" indirectly becomes the main factor of the satisfaction rating.

(4) The case where the evaluator $T_{2}$ gives $b_{1}$ to

"Kodama" (The main factor is "Nozomi")

The main factor of the shadow is "Nozomi" this time. Then the evaluator $T_{2}$ gives $b_{1}$ to "Kodama", $a_{1}$ and $c_{1}$ influence the evaluation value of $b_{1}$. Then, the size of the demand may be cut by $w_{23}=a_{1} c_{1}$, and then $w_{23}=a_{1} c_{1}$ is the satisfaction rating will rise among the valuators to "Kodama".

A similar interpretation is possible for "Hikari"and "Nozomi" of the Eq.8. The criterion matrix $W$ is a matrix of the satisfaction rating to the evaluation matrix $U$ when interpreted like

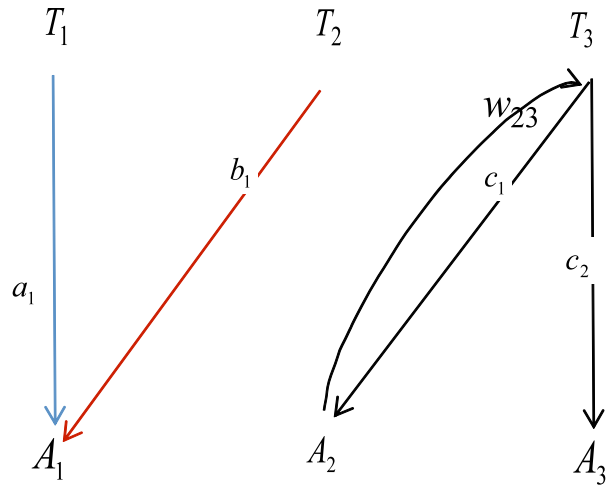

Kodama Hikari Nozomi this. Aassuming the evaluation value to evaluator's final alternatives to be $z_{1}, z_{2}$, and $z_{3}$, the overall evaluation of "Kodama" becomes $\left(a_{1} w_{11}+b_{1} w_{21}\right) z_{1}+a_{1} w_{12} z_{2}+b_{1} w_{23} z_{3}$ and is proportional to $z_{1}$.

\section{Example of application and knowledge to the ANP}

\subsection{Dilemma problem of Triantapyllou ${ }^{(4)}$}

Table 3 is a case where the order reversal is caused by pointing out by Tiantaphyllou,E. (2001). Such a dilemma problem is caused easily when there are a lot of criteria and alternatives.

Table 3. Order reversal by Triantapyllou

\begin{tabular}{|c|c|c|c|c|c|}
\hline & $\mathrm{C} 1(4 / 22)$ & $\mathrm{C} 2(9 / 22)$ & C3(9/22) & Evaluatio & hormalizt \\
\hline $\mathrm{A} 1$ & $9 / 9$ & $5 / 8$ & $2 / 8$ & 0.5398 & 0.2844 \\
\hline A2 & $1 / 9$ & $8 / 8$ & $5 / 8$ & 0.6850 & 0.3609 \\
\hline \multirow[t]{3}{*}{ A3 } & $8 / 9$ & $2 / 8$ & $8 / 8$ & 0.6730 & 0.3546 \\
\hline & A2> & A3 $>$ & A 1 & & \\
\hline & $\mathrm{C} 1(4 / 22)$ & $\mathrm{C} 2(9 / 22)$ & C3(9/22) & Evaluatio & Normalize \\
\hline A2 & $1 / 8$ & $8 / 8$ & $5 / 8$ & 0.6875 & 0.4979 \\
\hline \multirow[t]{2}{*}{ A3 } & $8 / 8$ & $2 / 8$ & $8 / 8$ & 0.6932 & 0.5021 \\
\hline & $\mathrm{C} 1(4 / 22)$ & $\mathrm{C} 2(9 / 22)$ & C3(9/22) & Evaluatio & hormalize \\
\hline $\mathrm{A} 1$ & $9 / 9$ & $5 / 8$ & $2 / 5$ & 0.6011 & 0.4176 \\
\hline \multirow[t]{2}{*}{ A2 } & $1 / 9$ & $8 / 8$ & $5 / 5$ & 0.8384 & 0.5824 \\
\hline & $\mathrm{C} 1(4 / 22)$ & $\mathrm{C} 2(9 / 22)$ & C3(9/22) & Evaluatio & hormalize \\
\hline $\mathrm{A} 1$ & $9 / 9$ & $5 / 8$ & $2 / 8$ & 0.6932 & 0.4856 \\
\hline \multirow[t]{2}{*}{ A3 } & $1 / 9$ & $8 / 8$ & $5 / 8$ & 0.7343 & 0.5144 \\
\hline & A3> & $\mathrm{A} 2>$ & A 1 & & \\
\hline
\end{tabular}


The main eigenvector $(0.2913,3696,3391)$ is obtained, when this method is applied to the dilemma problem of Triantapyllou. By the way, the main eigenvector, in one the criteria of the super-matrix when regularizing, becomes $(0.2818,3799,3382)$, and is not same as the previous eigenvector.

However, the ratio of the row is the same, even if Eq.(1) is regularized, and the value of A, B, and C of Eq.(2) doesn't change. This reason is as follows: The following equations are obtained from Eq.(5) for the main eigenvector; $\boldsymbol{W} \boldsymbol{z}=\boldsymbol{k} \boldsymbol{x}$ and $\boldsymbol{U} \boldsymbol{x}=k \boldsymbol{z}$. When $\boldsymbol{x}$ is deleted;

$$
\begin{aligned}
\boldsymbol{U W} z & =\left[\begin{array}{ccc}
a_{1} & b_{1} & 0 \\
a_{2} & 0 & c_{1} \\
0 & b_{2} & c_{2}
\end{array}\right] \cdot\left[\begin{array}{ccc}
\frac{b_{2} c_{1}}{a_{1} b_{2} c_{1}+a_{2} b_{1} c_{2}} & \frac{b_{1} c_{2}}{a_{1} b_{2} c_{1}+a_{2} b_{1} c_{2}} & 0 \\
\frac{a_{2} c_{2}}{a_{1} b_{2} c_{1}+a_{2} b_{1} c_{2}} & 0 & \frac{a_{1} c_{1}}{a_{1} b_{2} c_{1}+a_{2} b_{1} c_{2}} \\
0 & \frac{a_{1} b_{2}}{a_{1} b_{2} c_{1}+a_{2} b_{1} c_{2}} & \frac{a_{2} b_{1}}{a_{1} b_{2} c_{1}+a_{2} b_{1} c_{2}}
\end{array}\right] \cdot\left[\begin{array}{c}
z_{1} \\
z_{2} \\
z_{3}
\end{array}\right] \\
& =\left[\begin{array}{ccc}
1 & \frac{A B}{B+A C} & \frac{A B C}{B+A C} \\
\frac{C}{B+A C} & 1 & \frac{B C}{B+A C} \\
\frac{1}{B+A C} & \frac{A}{B+A C} & 1
\end{array}\right] \cdot\left[\begin{array}{c}
z_{1} \\
z_{2} \\
z_{3}
\end{array}\right]
\end{aligned}
$$

Eq.(8) is obtained. That is, the ratio between elements of the row is the same, even if the row of alternatives of the super-procession is regularized, and the value of A, B, and C don't change. However, it is not significant to the regularization of the row of the criterion of the super-matrix. Because there are six variables but only three equations when the row of the criterion of the super-procession is adjusted to one. These problems can be solved if the conditions are given. This means that $\mathrm{A}, \mathrm{B}$, and $\mathrm{C}$ become not independent any longer. That is, the meaning is not found in adjusting the row of the criterion of the super-matrix. Regularization has been said to be natural in the AHP. In the ANP, regularization is often available. However, the case where the reversal of the order of an integrated value is caused is often generated by regularization. The illustration of Triantapyllou suggests having not to regularize the criterion-matrix easily.

\subsection{Discussion of the dilemma or circulation problem with fallacy of composition}

A fallacy of composition arises when the whole is inferred from the fact that each choice is appropriate of some part of the whole. In recent years, the life cycle of the commodity is short, and the development risk has risen to, since the value chain reverses and the product development is indispensable based on customers' needs. Therefore, the decision making problem is important to solve in the enterprise.

For instance, let's assume the case where the evaluation of a certain product planning separates mutually in the farm enterprise and the development section and the production department like the satisfaction rating shown in Table 4. Let's apply the proposed method to this illustration. 
Table 4. Dilemma problem with fallacy of composition ${ }^{(3)}$

\begin{tabular}{|l|c|c|c|c|}
\hline & Farm & Development & Production & Total \\
\hline Commodity1 & 83 & 72 & 65 & 220 \\
\hline Commodity2 & 77 & 56 & 85 & 218 \\
\hline Commodity3 & 64 & 85 & 70 & 219 \\
\hline
\end{tabular}

The enterprise evaluates the order of Commodity1(83) $>$ Commodity2 (77) $>$ Commodity3 (64) and the development section evaluates the order of Commodity3(85)>Commodity1(72) $>$ Commodity2 (56). On the other hand, the evaluation of the production department evaluates the order of Commodity3(70) $>$ Commodity2(85)>Commodity1(65). Therefore a fallacy of composition arises. Then, evaluation matrix $\boldsymbol{U} \boldsymbol{W}$ is made based on Commodity1 in the first row, Commodity 2 in the second row and Commodity 3 in the third row.

$$
\boldsymbol{U} \boldsymbol{W}=\left[\begin{array}{ccc}
1 & 1.2857 & 0.9286 \\
0.9277 & 1 & 1.2143 \\
0.7711 & 1.5186 & 1
\end{array}\right]
$$

Moreover, the matrix is made by crossing the element of, each line of $\boldsymbol{U W}$.

$$
\left[\frac{A^{2} B^{2} C}{(B+A C)^{2}} \quad \frac{B C^{2}}{(B+A C)^{2}} \quad \frac{A}{(B+A C)^{2}}\right]=\left[\begin{array}{lll}
1.1939 & 1.1265 & 1.1704
\end{array}\right]
$$

By neglecting the denominator of the element, $A=1.1704 、 B=0.8769$ and $C=1.1334$ are obtained. Finally, the same shape of Eq. (1) is obtained. Then, we consider the eigenvector of the super-matrix based on the evaluation matrix whose element without the evaluation is 0 , by taking the inverse of the matrix as the evaluation matrix.

$$
\left[\begin{array}{cc}
\boldsymbol{0} & \boldsymbol{W} \\
\boldsymbol{U} & \boldsymbol{0}
\end{array}\right]=\left[\begin{array}{cccccc}
0 & 0 & 0 & 0.5144 & 0.3980 & 0 \\
0 & 0 & 0 & 0.4538 & 0 & 0.6020 \\
0 & 0 & 0 & 0 & 0.5312 & 0.3980 \\
1.1704 & 0.8769 & 0 & 0 & 0 & 0 \\
1 & 0 & 1.1334 & 0 & 0 & 0 \\
0 & 1 & 1 & 0 & 0 & 0
\end{array}\right]
$$

This eigenvector becomes $(0.3362,0.3298,0.3340)$.

By the way, if the evaluation value of the farm enterprise to Commodity1 doesn't change, an actual satisfaction rating to Commodity2 is higher than $83 / 1.1704=71$. Oppositely, if the evaluation value of the farm enterprise to Commodity 2 doesn't change, an actual satisfaction rating to Commodity1 is lower than $77 \times 1.1704=90$. In other words, there is room to review the evaluation because the satisfaction figures to be wide. Table 5 shows the width of the evaluation, correction value in the geometric mean, the sum, and ratio. The agreement will be necessary to revise the value of each such evaluation. 
Table 5. Review of evaluation value

\begin{tabular}{|l|c|c|c||c|c|c||c|c|}
\hline & \multicolumn{3}{|c||}{ Review } & \multicolumn{3}{c||}{ Mean Squere } & Sum & Ratio \\
\hline & Farm & Develop & Pro & Farm & Develop & Pro & Final & \\
\hline Com1 & $90 / 83$ & $75 / 74$ & & 86 & 74 & & 160 & 0.3397 \\
\hline Com2 & $77 / 71$ & & $79 / 85$ & 73 & & 81 & 154 & 0.3270 \\
\hline Com3 & & $86 / 85$ & $70 / 75$ & & 85 & 72 & 157 & 0.3333 \\
\hline
\end{tabular}

The obtained eigenvector $\mathrm{W}\left(w_{1}, w_{2}, w_{3}\right)$ is the geometric mean. This reason is thought as follows. Multiplied by the geometric mean of $\mathrm{B}$ and $\mathrm{C}$ is shown below.

$$
\begin{gathered}
{\left[\begin{array}{lll}
A^{2} B^{2} C & B C^{2} & A
\end{array}\right]=\left[\begin{array}{lll}
1.1939 & 1.1265 & 1.1704
\end{array}\right]=\left(\begin{array}{lll}
w_{1}{ }^{3} & w_{2}{ }^{3} & w_{3}^{3}
\end{array}\right)^{\prime}} \\
B=\frac{w_{1}{ }^{2}}{w_{2} w_{3}{ }^{4}} \text { and } C=\frac{w_{2}{ }^{2} w_{3}{ }^{2}}{w_{1}} \\
B C=\frac{w_{1} w_{2}}{w_{3}{ }^{2}}
\end{gathered}
$$

On the other hand, eigenvector $\boldsymbol{z}\left(z_{1}, z_{2}, z_{3}\right)$ and constant of proportion $\beta$ of $\boldsymbol{U} \boldsymbol{W}$ are used.

$$
\mathrm{z}_{1}=\frac{B\left\{(B+A C)\left(1-k^{2}\right)-A C\right\}}{(B+A C)\left(1-k^{2}\right)-B}=\frac{w_{1}}{\beta} \quad \mathrm{z}_{2}=\frac{C\left\{(B+A C)\left(1-k^{2}\right)-B\right\}}{(B+A C)\left(1-k^{2}\right)-A C}=\frac{w_{2}}{\beta}
$$

Because $\mathrm{z}_{1} z_{2}=B C=\frac{w_{1} w_{2}}{\beta^{2}}$ is equal to expression of Eq.(8), the constant of proportion becomes $\beta=w_{3}$.Therefore, $\mathrm{z}_{1}=\frac{w_{1}}{w_{3}} 、 \mathrm{z}_{2}=\frac{w_{2}}{w_{3}}$ and $\mathrm{z}_{3}=\frac{w_{3}}{w_{3}}$ are obtained, the eigenvectors obtained by this method is always a value of the geometrical mean.

\section{Conclusion.}

The following findings can be obtained from this paper.

(1) A new method to solve the dilemma or circulation problem is proposed by using the ANP, and the legitimate of which is mathematically proven.

(2) In the ANP, regularization is often available. However, the case where the reversal of the order of an integrated value is caused is often generated by regularization. The illustration of Triantapyllou suggests having not to regularize the criterion-matrix easily.

(3) The evaluation value is not absolute. In other words, there is room to review the evaluation because the satisfaction figures to be wide. The method is useful to revise the value of each such evaluation. 
This study was part of results of research by the 2008 Research Fellowship of the Nagoya Gakuin University. In addition, one of the authors got an opportunity of the long-term training from Taiwan and some profitable advice was obtained from the Distinguished Chair Professor Gwo-Hhiung Tzeng at the Kainan University. We wish to express our gratitude for adding it.

\section{Appendices}

\section{Calculating the eigenvector in the AHP}

Let's consider the following matrix of the dilemma or circulation problem.

$$
\boldsymbol{U}=\underset{\text { Hozomi } i}{\text { Kodama }}\left[\begin{array}{ccc}
a_{1} & b_{1} & 0 \\
a_{2} & 0 & c_{1} \\
0 & b_{2} & c_{2}
\end{array}\right]
$$

Pairs are compared from Eq. (A1), and the matrix is represented by A, B, and C.

$$
\boldsymbol{U}_{A H P}=\left[\begin{array}{lll}
a_{1} / a_{1} & a_{1} / a_{2} & b_{1} / b_{2} \\
a_{2} / a_{1} & a_{2} / a_{2} & c_{1} / c_{2} \\
b_{2} / b_{1} & c_{2} / c_{1} & c_{2} / c_{2}
\end{array}\right]=\left[\begin{array}{ccc}
1 & A & B \\
1 / A & 1 & C \\
1 / B & 1 / C & 1
\end{array}\right]
$$

Let's assume the maximum eigenvalue and the eigenvector of this matrix to be $\alpha$ and $\left(y_{1}, y_{2}, y_{3}\right)$.

$$
\begin{aligned}
& y_{1}+A y_{2}+B y_{3}=\alpha y_{1} \\
& y_{1} / A+y_{2}+C y_{3}=\alpha y_{2} \\
& y_{1} / B+y_{2} / C+y_{3}=\alpha y_{3}
\end{aligned}
$$

When Eq.(A4) from Eq.(A2) are arranged,

$$
\begin{gathered}
(1-\alpha) y_{1}+A y_{2}+B y_{3}=0 \\
y_{1} / A+(1-\alpha) y_{2}+C y_{3}=0 \\
y_{1} / B+y_{2} / C+(1-\alpha) y_{3}=0
\end{gathered}
$$

Eq.(A5) from Eq.(A7) are obtained. From Eq.(A5) and Eq.(A7),

$$
y_{1}=\frac{B\{A C(1-\alpha)-B\}}{B(1-\alpha)-A C} y_{3}
$$

from Eq.(A6) and Eq.(A7), 


$$
y_{2}=\frac{C\{B(1-\alpha)-A C\}}{A C(1-\alpha)-B} y_{3}
$$

are obtained. As $y_{3}=1$,

$y_{1}=\frac{B\{A C(1-\alpha)-B\}}{B(1-\alpha)-A C}$ and $y_{2}=\frac{C\{B(1-\alpha)-A C\}}{A C(1-\alpha)-B}$ are get as the eigenvector.

\section{Calculating the eigenvector in the ANP}

Next, let's think about super-matrix $\boldsymbol{U}$ based on the evaluation matrix $\boldsymbol{W}$ whose element without the evaluation is 0 .

$$
\boldsymbol{W}=\left[\begin{array}{ccc}
\frac{b_{2} c_{1}}{a_{1} b_{2} c_{1}+a_{2} b_{1} c_{2}} & \frac{b_{1} c_{2}}{a_{2} b_{2} c_{1}+a_{2} b_{1} c_{2}} & 0 \\
\frac{a_{2} b_{2} c_{1}+a_{2} b_{1} c_{2}}{a_{1}} & 0 & \frac{a_{1} c_{1}}{a_{1} b_{2} c_{1}+a_{2} b_{1} c_{2}} \\
0 & \frac{a_{1} b_{2}}{a_{1} b_{2} c_{1}+a_{2} b_{1} c_{2}} & \frac{a_{2} b_{1}}{a_{1} b_{2} c_{1}+a_{2} b_{1} c_{2}}
\end{array}\right]
$$

Let's assume the maximum eigenvalue and the eigenvector of this matrix to be $k$ and $\boldsymbol{x}$ and $\boldsymbol{z}$ in the

ANP.

$$
\left[\begin{array}{cc}
0 & W \\
U & 0
\end{array}\right]\left[\begin{array}{l}
x \\
z
\end{array}\right]=k\left[\begin{array}{l}
x \\
z
\end{array}\right]
$$

From $\boldsymbol{W} z=\boldsymbol{k} \boldsymbol{x}$ and $\boldsymbol{U} \boldsymbol{x}=\boldsymbol{k} \boldsymbol{z}, \boldsymbol{U W} \boldsymbol{z}=k^{2} \boldsymbol{z}$ is

$$
\begin{aligned}
\boldsymbol{U W z} & =\left[\begin{array}{ccc}
a_{1} & b_{1} & 0 \\
a_{2} & 0 & c_{1} \\
0 & b_{2} & c_{2}
\end{array}\right] \cdot\left[\begin{array}{ccc}
\frac{b_{2} c_{1}}{a_{1} b_{2} c_{1}+a_{2} b_{1} c_{2}} & \frac{b_{1} c_{2}}{a_{1} b_{2} c_{1}+a_{2} b_{1} c_{2}} & 0 \\
\frac{a_{2} c_{2}}{a_{1} b_{2} c_{1}+a_{2} b_{1} c_{2}} & 0 & \frac{a_{1} c_{1}}{a_{1} b_{2} c_{1}+a_{2} b_{1} c_{2}} \\
0 & \frac{a_{1} b_{2}}{a_{1} b_{2} c_{1}+a_{2} b_{1} c_{2}} & \frac{a_{2} b_{1}}{a_{1} b_{2} c_{1}+a_{2} b_{1} c_{2}}
\end{array}\right] \cdot\left[\begin{array}{l}
z_{1} \\
z_{2} \\
z_{3}
\end{array}\right] \\
& =\left[\begin{array}{ccc}
1 & \frac{a_{1} b_{1} c_{2}}{a_{1} b_{2} c_{1}+a_{2} b_{1} c_{2}} & \frac{a_{1} b_{1} c_{1}}{a_{1} b_{2} c_{1}+a_{2} b_{1} c_{2}} \\
\frac{a_{2} b_{2} c_{1}}{a_{1} b_{2} c_{1}+a_{2} b_{1} c_{2}} & 1 & \frac{a_{2} b_{1} c_{1}}{a_{1} b_{2} c_{1}+a_{2} b_{1} c_{2}} \\
\frac{a_{2} b_{2} c_{2}}{a_{1} b_{2} c_{1}+a_{2} b_{1} c_{2}} & \frac{a_{1} b_{2} c_{2}}{a_{1} b_{2} c_{1}+a_{2} b_{1} c_{2}} & 1
\end{array}\right] \cdot\left[\begin{array}{l}
1 \\
z_{1} \\
z_{2} \\
z_{3}
\end{array}\right]
\end{aligned}
$$

obtained. 


$$
\begin{aligned}
& \left(1-k^{2}\right)_{1}+\frac{a_{1} b_{1} c_{2}}{a_{1} b_{2} c_{1}+a_{2} b_{1} c_{2}} z_{2}+\frac{a_{1} b_{1} c_{1}}{a_{1} b_{2} c_{1}+a_{2} b_{1} c_{2}} z_{3}=0 \\
& \frac{a_{2} b_{2} c_{1}}{a_{1} b_{2} c_{1}+a_{2} b_{1} c_{2}} z_{1}+\left(1-k^{2}\right)_{2}+\frac{a_{2} b_{1} c_{1}}{a_{1} b_{2} c_{1}+a_{2} b_{1} c_{2}} z_{3}=0 \\
& \frac{a_{2} b_{2} c_{2}}{a_{1} b_{2} c_{1}+a_{2} b_{1} c_{2}} z_{1}+\frac{a_{1} b_{2} c_{2}}{a_{1} b_{2} c_{1}+a_{2} b_{1} c_{2}} z_{2}+\left(1-k^{2}\right) z_{3}=0
\end{aligned}
$$

From Eq.(A12) and Eq.(A14),

$$
z_{1}=\frac{\frac{b_{1}}{b_{2}}\left\{\left(1-k^{2}\right)-\frac{a_{1} b_{2} c_{1}}{a_{1} b_{2} c_{1}+a_{2} b_{1} c_{2}}\right\}}{\left(1-k^{2}\right)-\frac{a_{2} b_{1} c_{2}}{a_{1} b_{2} c_{1}+a_{2} b_{1} c_{2}}} z_{3} \text { is obtained. }
$$

From Eq.(A13) and Eq.(A14),

$$
z_{2}=\frac{\frac{c_{1}}{c_{2}}\left\{\left(1-k^{2}\right)-\frac{a_{2} b_{1} c_{2}}{a_{1} b_{2} c_{1}+a_{2} b_{1} c_{2}}\right\}}{\left(1-k^{2}\right)-\frac{a_{1} b_{2} c_{1}}{a_{1} b_{2} c_{1}+a_{2} b_{1} c_{2}}} z_{3}
$$

Here, when rewriting by $A, B, C$ and $1-\frac{1}{1-k^{2}}=\beta-1$ under the $z_{3}=1$,

$$
\begin{gathered}
z_{1}=\frac{\left.B\{B+A C)\left(1-k^{2}\right)-A C\right\}}{(B+A C)\left(1-k^{2}\right)-B}=\frac{B\{B-(1-\beta) A C\}}{A C-(1-\beta) B}=\frac{B\{(1-\beta) A C-B\}}{(1-\beta) B-A C}=y_{1} \\
z_{2}=\frac{C\left\{(B+A C)\left(1-k^{2}\right)-B\right\}}{(B+A C)\left(1-k^{2}\right)-A C}=\frac{C\{A C-(1-\beta) B\}}{B-(1-\beta) A C}=\frac{C\{(1-\beta) B-A C\}}{(1-\beta) A C-B}=y_{2}
\end{gathered}
$$

Therefore, the eigenvector of Eq.(A11) becomes equivalent with the eigenvector of $\boldsymbol{U}_{A H P}$.

\section{Literatures}

(1) Arrow,K,J. (1951). Social Choice and Individual Values, Yale University Pree.

(2) Sugiura,S., Kinoshita,E. (2005). Solution of Circular Logic by Concurrent Convergence Method of Evaluating Value, Urban Science Studies, Meijo University, 10,115-121.

(3) Sugiura,S., Kinoshita.E. (2007). A Dissolution of Circular Logic on Social Choice, Journal of Japanese Symposium on The Analytic Hierachy Proce,1,75-83.

(4) Tiantaphyllou,E. (2001). Two New Cases of Rank Reversals when the AHP and Some of its Additive 
Variants are Used that do not Occur with the Multiplicative AHP, J. Multi-Crit. Decis. Anal. 10, 1125 . 